\title{
Stripe formation in electron-doped cuprates
}

\author{
A. Sadori and M. Grilli \\ Istituto Nazionale di Fisica della Materia and Dipartimento di Fisica, Università di Roma "La Sapienza", \\ Piazzale A. Moro 2, Roma, Italy 00185
}

\begin{abstract}
We investigate the formation of charge domain walls in an electron-doped extended Hubbard model for the superconducting cuprates. Within an unrestricted Hartree-Fock approach, extended by the introduction of slave-bosons to obtain a more proper treatment of strong correlations, we demonstrate the occurrence of stripes in the $(1,1)$ and $(1,-1)$ directions having one doped electron per stripe site. The different filling, direction and width of these electron-doped stripes with respect to those obtained in the hole-doped systems have interesting observable consequences, which are discussed.
\end{abstract}

PACS: 71.10. w, 71.28.+d, 74.72. h, 71.45.Lr

The issue of charge inhomogeneity in the cuprates and particularly in the underdoped region of their phase diagram is presently attracting a great attention both from the theoretical and the experimental point of viewt.

Some theoretical proposals are based on the occurrence of stripe phases with the charge tending to order in incommensurate charge-density waves or in fluctuating domain walls to explain both the apomalous normal state properties and superconductivity a $^{2}$. In particular it was proposed 3 that the stripe phase in the underdoped cuprates is ruled by a charge-driven critical line ending in a hidden quantum critical point (QCP) located around optimal doping. Although this QCP may be masked by (strong) pairing due to the nearly singular attractive charge fluctuation 5 . 5 the physics of the underdoped cuprates is strongly influenced by the tendency to spatial charge order.

On the experimental side, the critical line for stripe formation has likely been defected in NQR $\mathrm{O}$ and in magnetotransport experiments 6 , where the suppression of superconductivity by strong magnetic fields both in holeand electron-doped materials reveal a metal-to-insulator crossover line ending at $T=0$ near optimal doping. In the hole-doped compounds the insulating character of the low-temperature and low-doping phase is stronger at the specific doping $1 / 8$, where commensurability effects between the charge ordering and the underlying lattice make the charge order particularly robust 6 . This latter effect is a signature of spatial order and provides further evidence of the enhanced strenght of stripe ordering in various compounds at specific fillings (this is the socalled $1 / 8$ phenomenon). This commensurability effect seems instead much less evident in the electron-doped materials.

There is also an increasing and more direct experimental evidence for the occurrence of (static or dynamis) charge ordering in under- and optimally dopedsupratest, ranging from EXAFS10 to neutron scattering 11 , and Xray scattering 22.13 .

The stripe instability was predicted theoretically in Ref. 14 within Hartree-Fock (HF) theory applied to the extended Hubbard model and confirmed by a number of subsequent investigation 15 . Only recently, however, the occurrence in the single-band Hubbard model of halffilled stripes (i.e. with half a hole per stripe site) in the $(1,0)$ [or equivalently in the $(0,1)$ ] direction [HalfFilled-Vertical stripes (HFVS)] was demonstrated within a generalized HF approach including slave bosons to suitably handle the strong local electron-electron interaction. This achievement remarkably showed that (a proper treatment of) strong correlations and long-range interactions are crucial in determining a specific stripe texture similar to the one observed in the cuprates.

The aim of the present work is to apply the same joynt HF-slave-boson technique to theoretically investigate the stripe formation in a more realistic extended three-band Hubbard model in the case of electronic doping. It is to be borne in mind that in any HF approach the solutions are self-consistently stabilized by locating the chemical potential in the middle of a gap. Therefore this approach is only suitable to describe insulating phases where the filling and/or the breaking of translational symmetry gives rise to such gaps. In our case we therefore intend to describe by our method the $1 / 8 \mathrm{com}-$ mensurate systems where both the charge ordering and the (nearly) insulating character are substantial.

- The model and the main outcomes. The starting point is the extended three-band Hubbard model

$$
\begin{aligned}
H & =\sum_{<i j>\sigma} t_{i j}^{p d}\left(d_{i \sigma}^{\dagger} p_{j \sigma}+h . c .\right)+\sum_{<j j^{\prime}>\sigma} t_{j j^{\prime}}^{p p}\left(p_{j \sigma}^{\dagger} p_{j^{\prime} \sigma}+h . c .\right) \\
& +\Delta \sum_{j \sigma} p_{j \sigma}^{\dagger} p_{j \sigma}+U_{d} \sum_{i} d_{i \uparrow}^{\dagger} d_{i \uparrow} d_{i \downarrow}^{\dagger} d_{i \downarrow} \\
& +U_{p} \sum_{j} p_{j \uparrow}^{\dagger} p_{j \uparrow} p_{j \downarrow}^{\dagger} p_{j \downarrow}+\sum_{i \neq j, \sigma \sigma^{\prime}} V_{i j} c_{i \sigma}^{\dagger} c_{i \sigma} c_{j \sigma^{\prime}}^{\dagger} c_{j \sigma^{\prime}}
\end{aligned}
$$

where the first and second terms describe the nearest neighbor (nn) copper-oxygen and oxygen-oxygen hopping of holes in the $d$ copper orbitals and in the $p$ oxygen orbitals respectively. $\Delta$ is the energy difference between $p$ and $d$ orbitals (the energy of the $d$ orbitals is set to zero), while $U_{d}\left(U_{p}\right)$ is the local Hubbard repulsion on the copper (oxygen) orbitals. The last term is the longrange interaction, with $V_{i j}=V e^{-r_{i j} / \lambda_{S}} /\left(2 r_{i j}\right)$, with $r_{i j}$ being the distance between two sites, $\lambda_{S}$ is the screening length. $V$ is set to $V=U_{p d} e^{1 / l_{S}}$ in order to match the 
$U_{p d} \mathrm{nn} \mathrm{Cu}-\mathrm{O}$ repulsion. In this term, the $c$ operators represent $d$ or $p$ hole operators depending on the nature of the site.

Following the procedure of Ref. 16 we treat the strong local repulsion $U_{d}$ by means of a slave-boson approach. The weaker strength of the repulsion on $p$ orbitals and the smaller average hole density on oxygen sytes makes unnecessary to apply the same technique to the oxygen orbitals. Therefore the other interaction terms $U_{p}$ and $V$ are treated by a standard HF decoupling.

We summarize here the main results valid both for hole and electron doping. i) Like in the single-band Hubbard model, also in the three-band Hubbard model the major effect of the slave-boson treatment is to stabilize the stripes 17 with respect to isolated spin polarons. This stability mostly arises because, according to general arguments 18 , the charge mobility tends to favor stripes with respect to isolated spin polarons. Then, since the Gutzwiller-like procedure implemented via the slave bosons allows the charge to delocalize without paying a much too large repulsive energy stripes are not artificially disfavored within our approach. The same is not true in standard HF treatments, where the double occupation of the sites is avoided via a strong antiferromagnetic polarization of the spins, which greatly suppresses the intersite hopping. ii) For moderate to large values of $U_{d}$, the natural energy scale for charge excitations no longer is $U_{d}$, but rather the charge-transfer energy $\Delta$ becomes the principal parameter determining the stability of stripes with respect to an ordered lattice (Wigner crystal) of maximally separated spin polarons. The stripes are only stable below a critical value $\Delta_{c}$, depending on the other parameters of the model, which in any reasonable realistic case is much larger than the critical values obtained in the standard (i.e. without slave-bosons) HF technique. iii) For a quite broad range of realistic values of the parameters the HFVS and the Filled Diagonal stripes [i.e. with one hole per stripe site and running along the $(1,1)$ or $(1,-1)$ directions (FDS)] are the most stable or the only stable solutions. In particular, for hole doping $x=1 / 8$, while the standard HF treatment favors the FDS, it was remarkably found that the HFVS become the ground state when strong correlations are properly treated by slave bosons. The absolute stability of HFVS occurs even in the absence of long-range interactions. In this respect, the hole-doped three-band Hubbard model seems more favorable than the single-band one in giving rise to half-filled stripe textures of the type observed in cuprates. iv) The local repulsion on oxygen only plays a minor role, while $\mathrm{v}$ ) the oxygen-oxygen hopping $t_{p p}$ enhances the charge mobility and therefore stabilizes the stripes with respect to the polaron Wigner crystal (POLWC). As far as the effects of the "long-range" interaction are concerned, it was found that a nn repulsion $U_{p d}$ naturally disfavors the stripes while it does not affect the POLWC, where the charges are at larger distance. Nevertheless vi) a moderate $U_{p d}$ stabilizes the HFVS with respect to the FVS, thus strengthening the stability of this configura- tion. On the other hand, vii) the large-distance part of a truely long range interaction turns out to increase the energy of the POLWC thus favoring further the stability of an array of stripes.

Finally, the effects of a coupling between holes and static lattice deformations were also investigated. In particular, the hopping was modulated by the breating displacement of oxygen ions in the $j$ site $t_{i j}\left(\left\{u_{j}\right\}\right)=$ $t_{p d} \pm \alpha u_{j}$, where the sign is positive if the $\mathrm{Cu}-\mathrm{O}$ distance is decreased, while it is negative if the $\mathrm{Cu}-\mathrm{O}$ distance increases. For simplicity, in this model we neglected the coupling between the $\mathrm{O}-\mathrm{O}$ hopping and the lattice. In an analogous way the orbital energy on the copper site is modified as $\epsilon_{i}\left(\left\{u_{k}\right\}\right)=\epsilon_{d}+\beta \sum_{j}\left( \pm u_{j}\right)$. Then the dimensionless hole-lattice coplings are $\lambda_{\alpha}=\alpha^{2} /\left(K t_{p d}\right)$ and $\lambda_{\beta}=\beta^{2} /\left(K t_{p d}\right)$. At variance with the findings of Ref. 19, where stripe textures were only found as local minima, the slave-boson treatment produces absolutely stable stripe solutions. However, owing to the specific choice of lattice displacement, there occurs a frustration effect whenever holes (or electrons) are located on neighboring sites. This frustration of the lattice displacement is not present in the case of distant polarons. Therefore in stripe configurations (particularly in the filled ones), viii) the electron-phonon coupling turns out to be detrimental for stripes in comparison to the POLWC. Nevertheless, the absolute stability of HFVS at hole-doping $x=1 / 8$ survives in the presence of a moderate coupling.

For concreteness, we report in Table I the energy difference between the HFVS or the FDS and the POLWC configurations at $x=1 / 8$ for a typical set of parameters in a $9 \times 8$ lattice. The HFVS stabilization effect of the long-range (LR) interaction is shown in the second line, while the destabilization due to the electron-phonon (EP) coupling is shown in the third line. The consideration of all the different interactions leads to a stable HFVS configuration as indicated in the fourth line. As shown in the last column, the FDS are instead always disfavored with respect to the POLWC.

\begin{tabular}{l|c|c} 
& $\epsilon_{H F V S}-\epsilon_{P O L W C}(\mathrm{eV})$ & $\epsilon_{F D S}-\epsilon_{P O L W C}(\mathrm{eV})$ \\
\hline $\mathrm{HSC}$ & -0.036 & +0.003 \\
$\mathrm{LR}$ & -0.084 & $?$ \\
$\mathrm{EP}$ & +0.009 & +0.034 \\
$\mathrm{EP}+\mathrm{LR}$ & -0.034 & $?$
\end{tabular}

Table I: Energy differences (per doped hole) between two different stripe configurations (HFVS and FDS) and the POLWC in a $9 \times 8$ (or $8 \times 8$ ) lattice with periodic boundary conditions. According to Ref. 20, the parameter values are $t_{p d}=1.3 \mathrm{eV}, t_{p p}=0.65 \mathrm{eV}, U_{d}=10.5 \mathrm{eV}, U_{p}=4 \mathrm{eV}$. The LR coupling is such that $U_{p d}=1.2 \mathrm{eV}$ with a screening length $\lambda_{S}=32$ lattice units. The EP couplings are $\lambda_{\alpha}=0.5$ and $\lambda_{\beta}=0$.

- Electron doping. While the above analysis was 
mostly carried out for hole doping (and particularly for $x=1 / 8$ ), it is quite important to extend the investigation to electron-doped systems. While the general validity of the above results is mantained, some important differences are also found. Specifically it was noticed that the charge in electron doped stripes is more localized. A comparison of the charge profile of hole-doped (HD) and electron-doped (ED) stripes is shown in Fig. 1, where the narrower charge distribution of ED stripes is apparent.
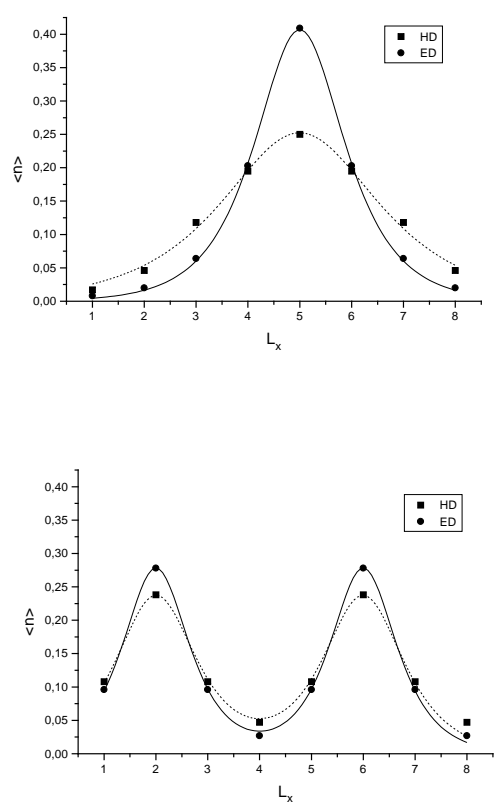

FIG. 1. Charge density distribution in the $(1,0)$ direction for FDS (i.e. in the (1,1) direction, above) and for HFVS (i.e. in the $(0,1)$ direction, below) for both ED and HD systems. The parameters are the same as in Table I with $x= \pm 1 / 8$, but with $\lambda_{\alpha}=\lambda_{\beta}=0$.

This effect is quite natural since the doped holes mostly reside on oxygen sites where due the smaller interaction and to the direct $t_{p p}$ hopping acquire a high mobility, while the doped electrons mostly reside on copper sites. Therefore the doped-electron mobility is lower because it only occurs via an intermediate charge transfer to oxygen sites. The smaller mobility and the consequent relative narrowness of the stripes in ED systems has two main consequences. The first is that the stripe formation is generically more difficult. Nevertheless stripe textures still remain the ground state for realistic values of the parameters. In particular, even in the presence of a sizable electron-lattice coupling (which, as discussed above, favors the POLWC solution more than the stripes) stable FDS textures are found. A second important effect is that FDS (with one doped electron per stripe site) become more stable than the HFVS. This latter finding suggests that ED cuprates should be more similar to the nickelates, where diagonal stripes with one doped hole per site are detected.

- Discussion. The above reported findings have important consequences. First of all, the fact that FDS accomodate one doped charge per site, rather than onehalf like the HFVS, increases the (average) distance between the stripes. For instance, at $|x|=1 / 8$, in the FDS case the stripes are at distance $d_{F D S}=8 a / \sqrt{2}$, while the HFVS are separated by $d_{H F V S}=4 a$. As a consequence the interstripe antiferromagnetically correlated background is better preserved when FDS are formed. This effect is emphasized by the narrow width of the ED stripes, which is naturally accompanied by a lower transversal mobility of the stripes. It should also be noticed, that static disorder effects due to pinning of the stripes should comparatively be stronger in ED materials, where the stripes are expected to be less mobile.

The formation of ordered arrays of stripes is naturally accompanied by an (incommensurate) spin response, which appears in neutron scattering at momenta displaced by an amount $\varepsilon$ from the AF wavevector $Q_{A F}=( \pm \pi / a, \pm \pi / a)$. While this effect has indeed been observed in metallic HD materials, with $\varepsilon \widetilde{2} x$ for $x$ up to $1 / 8$ and then saturating $(\varepsilon \sim$ constant $) 21$, comparatively detailed neutron scattering data are not available for ED systems. Our findings suggest that the incommensurate momenta should scale as $\varepsilon \sim x / \sqrt{2}$. Moreover, while in HD materials the spin excitation peaks appear in the $(1,0)$ and $(0,1)$ directions consistently with the vertical ordering of the stripes, in ED materials the corresponding peaks should be observed in the $(1,1)$ and $(1,-1)$ directions, according to our finding of diagonal stripe formation. However, the substantial disorder effects due to pinning could broaden the spin peaks making the observation of incommensurability more difficult. In this respect more local probes like the NQR (successfully applied in the HD materialst) can turn out to be very useful.

As far as transport is concerned, no theory is presently available in the case of scattering due to (dynamically fluctuating) stripes. Therefore no definite statement can be put forward beside the following generic remarks. The first generic observation is that the narrower charge distribution of stripes in ED systems, indicates a smaller transversal mobility. This, together with the larger separation of the filled ED stripes accounts for the more extended insulating region of the ED systems with respect to the HD ones. Specifically this could explain why the antiferromagnetic phase is quite more robust upon electron doping than upon hole doping stressing the analogy between the stripes in ED systems and in nickelates.

As far as the longitudinal (i.e. along the stripes) metallicity is concerned, it can be noticed that HFVS should be more metallic than FDS. This is expected because in the longitudinal direction HFVS are similar to an array of quarter-filled onedimensional wires. If these wires 
escape the longitudinal CDW commensurate instability, this 1D structures should naturally be moremetallic than 1D chains with an empty or a full band22. If this is the case, a mechanism for superconductivity based on coupled metallic chains 3 seems hardly applicable to the ED case at least at the low and intermediate doping regimes where (according to our calculations) the stripes are expected to be less mobile in the transverse direction, nearly insulating in the longitudinal direction, and far one from the other. Of course this objection no longer holds when, by increasing doping, the metallic character of the system increases and our calculational scheme is no longer applicable. In this regime the stripes will be closer and likely far from the completely filled condition. In this latter doping region, however, the proximity to the quantum critical point observednear $x=0.179$ opens the way to alternative mechanisms 2 based on the scattering from critical charge fluctuations.

The metallic properties of ED stripes should also be strongly affected by disorder effects, which, as already mentioned above, are expected to be more important when the stripesare less mobile. In this regard, the recent observation 23 that small amounts of interstitial apical oxygen suppress superconductivity (but not metallicity) giving rise to pseudogap effects is of obvious relevance. A natural speculation could be that the interstitial oxygen triggers a pinning of the stripes. The suppression of stripe fluctuations then induces the suppression of superconductivity giving rise to a metallic (nonsuperconducting) state with disordered static (and locally well-formed) stripes with the consequent stronger reduction of density of states near the Fermi level.

Another generic consequence is that the diagonal stripes of the ED systems, should display rather different commensurability effects than in the HD materials. This is a consequence of the different spacing and ordering direction as well as of the different $T^{\prime}$ crystal structure of the ED cuprates. In particular, in the ED case, the 1/8 effects are not expected to be marked 4 since the stripes are quite far apart and the collective nature of the ordered state is likely affected by disorder pinning.

In conclusion, the occurrence of stripes in a rather realistic model for ED cuprates is a quite intriguing finding. According to our results, the specific features of the stripes in ED material are different from the features of stripes in HD ones. Therefore, if the occurrence of stripes in electron-doped systems will be experimentally confirmed, the comparison with the stripes already observed in the hole-doped materials will likely shed light on the physical mechanisms acting in both ED and HD cuprates. This calls for a search of experimental signatures and characterization of stripes in ED cuprates, hopefully leading to a better understanding of the cuprates.

Acknowledgments We gratefully acknowledge stimulating discussions with C. Castellani and C. Di Castro. The interaction with G. Seibold has been interesting and technically very helpful.
${ }^{1}$ For a general review on stripe experiments see Proceedings of the Second International Conference on Stripes and High- $T_{c}$ Superconductivity, June 2-6, 1998, Rome, Italy, [J. Supercond. (to be published)].

${ }^{2}$ C. Castellani, C. Di Castro, and M. Grilli, Phys. Rev. Lett. 75, 4650 (1995); A. Perali, C. Castellani, C. Di Castro, and M. Grilli, Phys. Rev. B 54, 16216 (1996).

${ }^{3}$ V.J. Emery, S.A. Kivelson, and O. Zachar, Phys. Rev. B56, 6120 (1997).

${ }^{4}$ J. Zaanen, O. Y. Osman, and W. van Saarlos, Phys. Rev. B 58, 11868 (1998).

${ }^{5}$ C. Castellani, C. Di Castro, and M. Grilli, Z. Phys. B, 103, 137 (1997).

${ }^{6}$ C. Castellani, C. Di Castro, and M. Grilli, J. Phys. Chem. Solids 59, 1694 (1998).

${ }^{7}$ A. W. Hunt, et al. Phys. Rev. Lett. 82, 4300 (1999).

${ }^{8}$ G. S. Boebinger, et al., Phys. Rev. Lett. 77, 5417 (1996).

${ }^{9}$ P. Fournier, et al., Phys. Rev. Lett. 81, 4720 (1998).

${ }^{10}$ A. Bianconi, Proceedings of the workshop on Phase Separation In Cuprate Superconductors, edited by K. A. Müller and G. Benedek (World Scientific, Singapore, 1993). For more recent EXAFS experiments on charge inhomogeneities see A. Bianconi, et al. Phys. Rev. Lett. 76, 3412 (1996).

11 J.M. Tranquada, J.D. Axe, N. Ichikawa, A.R. Moodenbaugh, Y. Nakamura, and S. Uchida, Nature (London) 375, 561 (1995).

12 A. Bianconi, et al., Phys. Rev. B 54, 12018 (1998).

${ }^{13}$ T. Niemöller, et al., cond-mat/9904383.

14 J. Zaanen and O. Gunnarson, Phys. Rev. B40, 7391 (1989).

${ }^{15}$ D. Poilblanc and T. M. Rice, Phys. Rev. B39, 9749 (1989); M. Kato, K. Machida, H. Nakanishi, and M. Fujita, J. Phys. Soc. J. 59, 1047 (1990); M. Inui and P. B. Littlewood, Phys. Rev. B44, 4415 (1991); H. J. Schulz, Phys. Rev. Lett. 641445 (1990); J. Zaanen and P. B. Littlewood, Phys. Rev. B50, 7222 (1994);

${ }^{16}$ G. Seibold, C. Castellani, C. Di Castro, and M. Grilli, Phys. Rev. B 58, 13506 (1998).

17 G. Seibold, E. Sigmund, and V. Hizhnyakov, Phys. Rev. B 57, 6937 (1998).

18 J. Zaanen and A. M. Oleś, Ann. Physik 5, 224 (1996).

19 Z. G. Yu, J. Zhang, J. T. Gammel, and A. R. Bishop, Phys. Rev. B 57, R3241 (1996).

${ }^{20}$ M. S. Hybertsen, M. Schlüter, and N. E. Christensen, Phys. Rev. B 39, 9028 (1989).

${ }^{21}$ In particular, at $x=1 / 8$ this is consistent with HFF separating antiphase spin domains with a periodicity $8 a 11$.

${ }^{22}$ Moreover, the effective intrastripe hopping is larger in the vertical $(0,1)$ than in the diagonal $(1,1)$ direction.

${ }^{23}$ Y. Onose, et al., Phys. Rev. Lett. 82, 5120 (1999)

${ }^{24}$ Nevertheless, a careful inspection of Fig. 3 in Ref. 9 , seem to indicate that a slight enhancement of the metalinsulator crossover temperature takes place for the sample at $x=0.13 \approx 1 / 8$. 